\title{
Agents, Believability and Embodiment in Advanced Learning Environments Introduction to a Panel Discussion
}

\author{
Anton Nijholt \\ University of Twente, PO Box 217 \\ 7500 AE Enschede, the Netherlands \\ anijholt@cs.utwente.nl
}

\begin{abstract}
On the world-wide-web we see a growing number of general HCI interfaces, interfaces to educational or entertainment systems, interfaces to professional environments, etc., where an animated face, a cartoon character or a human-like virtual agent has the task to assist the user, to engage the user into a conversation or to educate the user. What to say about the effects a human-like agent has on a student's performance? We discuss agents, their intelligence, embodiment and interaction modalities. In particular we introduce viewpoints and questions about roles embodied agents can play in educational environments.
\end{abstract}

\section{Introduction}

This short paper is meant to introduce the issues that underlie the introduction of embodied agents in learning environments. Embodied agents appear in different forms. We can just have a simple 2D talking face or a cartoonlike human figure on a web page or in a separate window making suggestions to the user, a desktop virtual reality environment where we have $3 \mathrm{D}$ avatars representing tutors or other learners or we can have an immersive Cave-like virtual reality environment where we can really experience interaction with a tutor, with objects and with other learners.

\section{Systems, Agents and Intelligence}

Before zooming in on some examples of embodied agents in learning environments it is useful to say something about the impact of computer systems in general, the impact of intelligent agent-like systems, the impact of believability, trustworthiness, emotion and personality modeling, and the impact of animated and life-like characters on the behavior of a human user of the interface or system.

Systems as Social Actors: Experiments have shown that when users engage with computer systems they at- tribute human characteristics to these systems. Not much intelligence has to be included in order to see this effect. Humans engage in social behavior toward computers. Studies and experiments show that users apply politeness norms to computers, they respond to computer personalities in the same way they respond to human personalities, they are susceptible to flattery and they apply gender stereotypes to computers (see e.g. Reeves \& Nass [13]).

Intelligent Software Agents: When we really aim at making a system more intelligent, as, for example, in intelligent tutoring systems, we may expect that apart from influencing the social behavior of the student toward the system, we have of course possibilities to steer a student's learning behavior, but also the student's cooperative, or motivational attitude can be influenced. This is even more true when we present the system or the interface to the system as some kind of actor (tutor) that knows, that reasons, that communicates and that displays consistent behavior in its environment. Agent technology is a research field that emerged in the 1990's and that can be considered as a field in which exactly such actors have to be developed, although not necessarily in the context of human-computer interaction. There have been a lot of discussions about what is exactly an agent and is not every computer program an agent. Some researchers explain that the answer is no (see e.g. Franklin \& Graesser [4]), other researchers have a pragmatic view: does the agent point of view helps us to develop ideas, helps us to become aware of possibilities and does it help us to communicate ideas. We don't think it is wise to underestimate the value of a good metaphor. Without going into details and especially controversial details, we want to mention properties of software modules that are generally assumed to be present before being allowed to talk about them as agents: autonomy, reactive and proactive behavior and the ability to interact with other agents (or humans). For an agent to act appropriately in a domain it has been useful to have an internal model in which we distinguish beliefs (what the agent regards to be true, this may change in time), desires (the goals the agent has committed himself to) and intentions (short-term plans that it tries to execute). 
Interacting Personalities: Software shows itself to the (human) user in the interface. This interface, whatever its form, may aggressively push information, it may try to pull information from a user, it may try to sell, to cheat, to seduce, to persuade, to flatter, etc. We need to mention the notions of believability, trustworthiness and emotions. Believability is an important notion that has been emphasized by Joseph Bates in the early 1990's. An agent is called believable, if some version of a personality shows in the interaction with a human. It does not necessarily mean that the agent is embodied although it is certainly true that in designing believable agents much can be learned from character-based artists that develop animate characters. In (Loyall [8]) requirements for believable agents have been investigated and attempts are given to fulfill these requirements. The main requirements are: personality, emotion, self-motivation, change, social relationships, consistency of expression and, finally, a list of properties that help to create the illusion of life in an agent (reactive and responsive, situatedness, appearance of goals, etc.). Trustworthiness is an other issue. How does a system show its good will and does it build credibility? In a text-based system face-to-face interaction cues (facial expressions, gestures, intonation, posture and gaze) are not available.

Embodied Agents: Now that we have discussed social, intelligent and believable behavior, it is time to consider the role of embodiment. Do we need embodiment to display the previously mentioned kinds of behaviors and when we assume embodiment of an agent, what is the extra impact of this behavior, how does this show in the agent's activities, and not less important, how can we use the embodiment as a multimedia modality to show information (e.g., the sequence of actions to handle complex machinery), to support verbal communication, and to display nonverbal behavior of the agent? Several authors have investigated nonverbal behavior among humans and the role and use of nonverbal behavior to support humancomputer interaction. See e.g. (Cassell [2]) for properties and impact of embodied conversational agents (with an emphasis on coherent facial expressions, gestures, intonation, posture and gaze in communication).

\section{Agents, Embodiment and Learning}

In the previous sections we surveyed developments in computer science (artificial intelligence, agent technology and graphics) that make it possible to talk about software modules and use them in application domains as agents and as embodied agents that can take the form of a $2 D$ or 3D talking face or an animated human-like body. Such agents are finding their way in learning environments. Are they pushed by the technology, are learners - having become accustomed to them in computer games - asking for them or do we have careful considerations about their use and careful experiments that evaluate their effectiveness in learning environments? And when we agree they can be effective, where and how to use them in a continuum between a constructivist and an instructionist approach? How should be their relation with teaching strategies such as tutoring, coaching, cognitive apprenticeship or Socratic dialogue? These questions need to be asked and answered. When we look at the current literature and survey the systems that have been designed and implemented in such a way that they allow experiments, two observations can be made First of all, several impressive research systems employing animated pedagogical agents have been built (see section 4). Secondly, and not surprisingly, we must observe that the abundance of ideas and technological possibilities, the multi-disciplinarity that is required and the lack of resources to have really comprehensive research programs that involve both advanced technology and large-scale empirical study, have not made it possible to give textbook-like decisive answers on how to use animated pedagogical agents, in what situations, and to achieve what goals. Nevertheless, with the observations on the abilities of animated agents in the previous section it is not difficult to predict that researchers will employ these agents in their systems.

Animated pedagogical agents have particular competence. As a real teacher they can show how to manipulate objects, they can demonstrate tasks and they can employ gesture to focus attention. As such they can give more customized advice in a rich learning environment, probably leading to improved problem solving by the student. Lester et al. [7] use the term deictic believability for agents that are situated in a world that they co-inhabit with students and in which they use their knowledge of the world, their relative location and their previous actions to create natural deictic gestures, motions, and utterances. There are more possibilities using animated agents to broaden the bandwidth of tutorial communication. When the agents are sufficiently expressive they can increase the student's enjoyment of the learning experience and the student's motivation. An agent can be designed for emotive believability, showing contextually appropriate facial expressions and expressive movements, not only to support and enhance the communication but also emotion (appreciation, enthusiasm, concern, disagreement, etc.) appropriate to the context. Encouragement, avoiding a student's frustration, conveying enthusiasm and making learning more fun are benefits that are mentioned when discussing the possibility to endow agents with emotive behavior and hence making it an interacting personality. As a result, students may spend more time using the (constructivist) learning environment, but also, as has been reported, there is a positive effect on student's perception of their learning experience. Such animated 
agents stimulate reflection and self-explanation and have a strong motivational effect. In Moreno et al. [9] a detailed report, including results on retention (recall of factual knowledge), problem-solving transfer (the ability to solve new problems based on similar principles) and motivation and interest, obtained by comparing learning in an animated agent-based environment with learning in a computer-based text environment, can be found.

\section{Embodied Agents: Learning Environments}

We mention some projects that we think are illustrative for the work on embodied agents in educational environments. We would like to mention the Soar Training Expert for Virtual Environments (STEVE, see Johnson et al. [6]) as an example of an advanced immersive 3-D learning environment with a virtual animated agent. In STEVE an animated, 3D, pedagogical agent gives instruction in procedural tasks in an immersive virtual environment. STEVE is able to demonstrate and explain a sequence of actions, monitor the movements and manipulations of the user, comment on them and suggest possible continuations to complete a task. In the JACOB project [3] a 3D agent walks and grasps objects in a particular order to help students how to solve the problem of the Towers of Hanoi. The student interacts by performing actions as well as by using natural language. The 'DesignA-Plant' project [7] is an interactive learning environment in which Herman the Bug acts as an agent that helps student to learn about plants and their environment. Especially this project has been subject of careful experiments concerning constructivist learning yielding very interesting results. AutoTutor $[5,12]$ is another tutoring system that uses NL dialogues for tutoring. The dialogue is delivered using an animated agent. Intonation and facial expressions of the talking head have been incorporated in order to present affective responses.

\section{Conclusions and Discussion}

We surveyed developments in computer science (artificial intelligence, agent technology and graphics) that make it possible to talk about software modules and use them in application domains as agents and even embodied agents that can take the form of a $2 \mathrm{D}$ or $3 \mathrm{D}$ talking face or an animated human-like body. Such agents are finding their way in learning environments. Are they pushed by the technology, are learners - having become accustomed to them in computer games - asking for them or do we have careful considerations about their use and careful experiments that evaluate their effectiveness in learning environments? And when we agree that they can be effective, where and how to use them in a continuum between a constructivist and an instructionist approach?
How should be their relation with teaching strategies such as tutoring, coaching, cognitive apprenticeship or Socratic dialogue? These questions need to be asked and answered. When is it worth the trouble? Human-like agents raise expectations. The learner expects human-like concern, social and competent behavior whatever he or she as learner is doing, etc. Isn't possible to increase the effect of computer-based learning environments without getting involved with creating models of emotion and personality of artificial embodied agents? Enough topics and approaches have been mentioned here to make a fruitful discussion possible.

\section{References}

[1] A.L. Baylor. Beyond butlers: Intelligent agents as mentors. Journal of Educational Computing \& Research. To appear.

[2] J. Cassell et al. (eds.). Embodied Conversational Agents. MIT Press, Cambridge, 2000.

[3] M. Evers \& A. Nijholt. Jacob, an agent for instruction in VR environments. Education and Information Technologies, T.A. Mikropoulos \& I.D. Selwood (eds.). to appear.

[4] S. Franklin \& A. Graesser. Is it an agent, or just a program? 3rd Intern. Workshop on Agent Theories, Architectures, and Languages, Springer, Berlin, 1996.

[5] A.C. Graesser et al. AutoTutor: A simulation of a human tutor. J. of Cognitive Systems Research. 1: 35-51, 1999.

[6] W. L. Johnson et al. Animated Pedagogical Agents: Face-toFace Interaction in Interactive Learning Environments. Intern. J. of Artificial Intelligence in Education (2000) 11, 47-78.

[7] J.C. Lester et al. Deictic and emotive communication in animated pedagogical agents. In: Cassell et al., 2000.

[8] A.B. Loyall. Believable Agents: Building interactive Personalities. CMU-CS-97-123, Carnegie Mellon University.

[9] R. Moreno. Life-like pedagogical agents in constructivist multi-media environments. EDMEDIA 2000, 741-746.

[10] A. Nijholt \& H. Hondorp. Towards communicating agents and avatars in virtual worlds. Proc. EUROGRAPHICS 2000, A. de Sousa \& J.C. Torres (eds.), August 2000, Interlaken, 91-95

[11] A. Paiva \& C. Martinho. A Cognitive Approach to Affective User Modeling. Proc. Affect in Interactions, Siena, 1999.

[12] N. Person et al. The integration of affective responses into AutoTutor. In [11].

13] B. Reeves \& C. Nass. The Media Equation. New York, Cambridge University Press, 1996. 